\title{
COSMIC RAY POWERED FOUNTAINS AND WINDS
}

\author{
H.J. VÖLK \\ Max-Planck-Institut für Kernphysik, P.O. Box 103980 \\ D-6900 Heidelberg, West Germany
}

ABSTRACT. The dynamics of galactic halos is discussed with special emphasis on the role of Cosmic Rays. Whereas Cosmic Rays should at best play a minor role for galactic fountain flows they appear essential in driving a wind from galaxies like ours. Active galaxies with a very hot interstellar medium $\left(T \geq 10^{7} \mathrm{~K}\right)$ have winds due to the thermal gas alone.

\section{INTRODUCTION}

Let me start by changing the title of this talk and cross out fountains from the title. This is because I do not believe that Cosmic Rays (CRs) play an important role in powering Galactic fountains. And I will give the corresponding physical arguments in section 2.3 below.

The question of the dynamics of the Galactic halo, especially that of winds and fountains has been discussed mostly without regard to CRs (e.g. Mathews and Baker, 1971; Habe and Ikeuchi, 1980; Kahn, 1981; Cox, 1981). These considerations included as an essential ingredient the radiative cooling of the thermal gas in a gravitational field that is determined by the stars of total mass $\mathbf{M}_{*}$ and, sometimes, in addition, by a distributed dark matter halo (with a mass $\mathrm{M}_{\mathrm{Halo}} \approx 3 \mathrm{M}_{*} \approx 8 \times 10^{11} \mathrm{M}_{\Theta}$ ). Assuming a spatially uniform disk, thermally driven winds exist only if the gas temperature in the disk is high enough, roughly, if $\mathrm{T}_{\text {disk }}$ exceeds $4.10^{6} \mathrm{~K}$, and $8.10^{6} \mathrm{~K}$ at a galactocentric distance of $12 \mathrm{kpc}$, and $1 \mathrm{kpc}$, respectively. This holds for the case of a massive dark halo. Without a dark matter halo the minimum disk temperatures are smaller by about a factor of 2 . For lower temperatures of gas, injected at disk midplane, the gas cools radiatively and falls back to the disk. This is the so-called Galactic fountain as described in the original paper by Shapiro and Field (1976).

If the disk gas is allowed to be nonuniform, both spatially and temporally (e.g. Norman and Ikeuchi, 1989, and references therein), then the energy input can be taken as correlated, as is for instance the case for Type II-SN explosions in OB associations (Heiles, 1984, 1989; Kennicutt, Edgar, and Hodge, 1989). The corresponding bubbles of hot gas can break through the extended neutral disk gas (Lockman, Hobbs, and Shull, 1986) into the halo which I define here as the region of fully ionised gas, commencing on average at a height $|\mathrm{z}| \approx 1 \mathrm{kpc}$ above (and below) the disk midplane. A given energy input and mean disk gas density can either lead to winds, i.e. gas secularly escaping from the galaxy, or to fountains or to both, at different positions. For our Galaxy at the present epoch, Norman and Ikeuchi prefer the fountain alternative. 


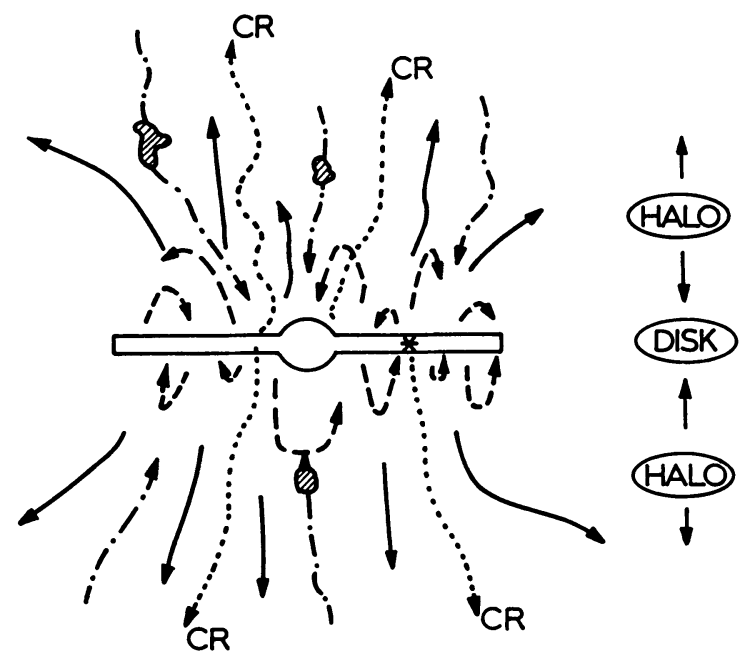

Figure 1: The various halo flows above the disk of a galaxy are shown schematically: Gas moving upwards and falling back to the disk, the fountains (dashed curves); hot gas moving secularly away from the disk, galactic wind (solid lines); infall of circum- or extragalactic matter (dashed-dot lines) in the form of "clouds" (hatched); CRs escaping the galaxy (dotted curves).

These are not the only possible flows in the halo of a galaxy (Figure 1): material might, in addition, fall in from large distances "outside" the galaxy (e.g. Mirabel, 1989), and finally the nonthermal gas component, the CRs, escapes the Galaxy. The latter fact and its consequences will be the main subject of this talk.

\section{2. "STATIC" HALO INCLUDING COSMIC RAYS}

\subsection{THE NONTHERMAL CR COMPONENT OF THE INTERSTELLAR MEDIUM.The}

CRs constitute the nonthermal, to a large extent even relativistic component of the Interstellar Medium (ISM). The dynamically relevant CRs with (individual particle) energies $\leq 10^{15} \mathrm{eV}$ probably originate at the shocks of Supernova Remnants (SNRs). These can convert some 10 to 30 percent of the total hydrodynamic energy released in a Supernova explosion into relativistic nucleons (Drury, Markiewicz, and Völk, 1989) essentially independent of the conditions of the ambient ISM (Markiewicz, Drury, and Völk, 1990). Within a factor of a few Supernovae thus appear to be standard candles for CRs wherever they explode! For our Galaxy these particles then populate the ISM for timescales (at energies $\mathrm{E}$ of a few $\mathrm{GeV} /$ nucleon) $\mathrm{t}_{\mathrm{esc}} \leq 10^{8} \mathrm{yr}$, decreasing with increasing $E$, before they escape. For the CR pressure $\mathrm{p}_{c}$ we have $\mathrm{p}_{\mathrm{c}} \approx 3.10^{-13}$ 
$\mathrm{dyn} / \mathrm{cm}^{2} \leq \mathrm{p}_{\mathrm{g}} \approx 6.10^{-13} \mathrm{dyn} / \mathrm{cm}^{2}$, where $\mathrm{p}_{\mathrm{g}}$ is the pressure of the thermal gas in the disk. (This value for $\mathrm{p}_{\mathrm{c}}$ is about a factor of 3 smaller than the one used by de Boer (1990) in the preceding talk). The average CR energy flow leaving the disk of the Galaxy is $F_{c o} \approx 10^{41} \mathrm{erg} / \mathrm{sec}$, roughly equal to a tenth of the total energy input from Galactic Supernovae. Since the mean random velocities of the CR particles approach the speed of light, their scale height $h_{c}$ as a gas tends to exceed $h_{g}$, the scale height of the thermal gas. In sharp contrast to the gas, CR nucleons suffer essentially no radiative cooling. Compared to typical spatial scales of the Galactic disk, their gyroradii are minute of order $10^{-6} \mathrm{pc}$ at $\mathrm{E}=5 \mathrm{GeV}$ in a $5 \mu \mathrm{G}$ magnetic field; they can escape only along magnetic field lines. Thus the conclusion is clear: the field lines must be partially open to extragalactic space. The question that remains is whether this escape is "bloodless", or whether the CRs drag some of the gas with them! The answer to this question depends on the transport properties of CRs. Their observed near-isotropy in momentum space shows that the particles are well scattered on fluctuations (waves) of the magnetic field $\underline{B}$ leading to spatial diffusion in the wave frame. This implies strong momentum coupling to the gas. Therefore, we can now rephrase the above question in an alternative form: is the gas dragged away by the CRs, at least at great heights in the halo, or do the CRs merely diffuse and drift (with the waves) away through the static gas (static halo)? Let me first discuss static halos.

2.2 STATIC COSMIC RAY HALOS (LOW DENSITY GAS) Dynamically selfconsistent static CR halos with selfexcited magnetic fluctuations and adiabatic thermal gas have been considered by Dougherty, McKenzie, and Westergaard (1985), and by Ko, Dougherty, and McKenzie (1990). These authors confined themselves to a plane halo geometry with straight field lines $\underline{B}$ = const. perpendicular to the plane of the Galactic disk. Ko et al. find that CR diffusion is negligible compared to Alfvénic drift as long as the (Rosseland) mean diffusion coefficient $\bar{k} \leq 10^{29} \mathrm{~cm}^{2} \mathrm{sec}^{-1}$, a number which has traditionally been considered as an upper bound for kinematic, purely diffusive and static CR halos (see e.g. the review by Ginzburg and Ptuskin, 1985). Thus diffusion in the wave frame can be neglected and then, as already shown by Dougherty et al., the dynamical equations for $\mathrm{p}_{\mathrm{g}}, \mathrm{p}_{\mathrm{c}}, \rho$, and $<(\delta \underline{B})^{2}>$ can be integrated analytically; here $\rho$ and $<(\delta \underline{B})^{2}>$ denote the mass density and mean square magnetic fluctuation amplitude, respectively. It is interesting that this strong coupling solution can actually be generalized to any open magnetic field configuration with arbitrary flux tube crossection $A(z)$, in particular to a halo that becomes spherically symmetric at distances large compared to the disk radius. I will not pause here for the proof but simply give a typical result (independent of $\mathrm{A}(\mathrm{z})$ in Table 1), where the suffices $\infty$ and o denote quantities for $|\mathrm{z}|=\infty$, and $|\mathrm{z}|=$ $1 \mathrm{kpc}$ at galactocentric distances $R_{\mathrm{O}}$. The quantitiy $\mathrm{p}_{\mathrm{w}}$ denotes the wave pressure $<(\delta \underline{B})^{2}>/ 8 \pi$ : The parameters at $|\mathrm{z}|=1 \mathrm{kpc}$ were chosen corresponding to a hot interstellar medium: $\mathrm{n}_{\mathrm{o}}=$ $3.10^{-3} \mathrm{H}$-atoms $/ \mathrm{cm}^{3}, \mathrm{p}_{\mathrm{go}} / \mathrm{K}=4000 \mathrm{~K} / \mathrm{cm}^{3}, \mathrm{p}_{\mathrm{co}}=2.7 \times 10^{-13} \mathrm{dyn} / \mathrm{cm}^{2}, \mathrm{~B}_{\mathrm{o}}=1 \mu \mathrm{G}$. The escape speeds to infinity at the galactocentric distances $R_{0}=1$, and $10 \mathrm{kpc}$ were taken as $\mathrm{u}_{\mathrm{esc}}(\mathrm{z}=\infty)=420$, and $600 \mathrm{~km} / \mathrm{sec}$, respectively. 
Thus, even if we were to assume that the dominant wave pressure $p_{w}$ could ultimately be dissipated away by some nonlinear damping or mode coupling process, the ratio $\mathrm{p}_{\mathrm{c}_{\infty}} / \mathrm{p}_{\mathrm{co}}$ is quite large and probably too large to be in equilibrium with the average intergalactic pressure. However $\mathrm{p}_{\mathrm{c} \infty}$ is presumably quite a bit lower than the total pressure in gas-rich clusters of galaxies. Since the gas should be fully ionized at $|z|>1 \mathrm{kpc}$ by OB-stars, halo and globular stars, and quasars (therefore assuring strong momentum coupling of gas and CRs) we expect such static halos to normally give way to winds except in clusters with high pressure hot intracluster gas.

\section{TABLE 1}

Gas pressure $p_{g \infty}, C R$ pressure $p_{c \infty}$, and wave pressure $p_{w \infty}$ at infinity for a static CR halo, normalised to the values $\mathrm{p}_{\mathrm{go}}=5.5 \times 10^{-13} \mathrm{dyn} / \mathrm{cm}^{2}$ and $\mathrm{p}_{\text {co }}=2.7 \times 10^{-13} \mathrm{dyn} / \mathrm{cm}^{2}$ at the reference level $|\mathrm{z}|=1 \mathrm{kpc}$, for two galactocentric distances $R_{0}=1$ and $10 \mathrm{kpc}$ in the disk.

\begin{tabular}{|c|c|c|c|}
\hline & $\mathrm{p}_{\mathrm{g} \infty} / \mathrm{p}_{\mathrm{go}}$ & $\mathrm{p}_{\mathrm{c} \infty} / \mathrm{p}_{\mathrm{co}}$ & $\mathrm{p}_{\mathrm{w} \infty} / \mathrm{p}_{\mathrm{co}}$ \\
\hline $\mathrm{R}_{\mathrm{o}}=1 \mathrm{kpc}$ & $10^{-4}$ & $2.8 \times 10^{-2}$ & $8 \times 10^{-2}$ \\
\hline $\mathrm{R}_{\mathrm{o}}=10 \mathrm{kpc}$ & $3 \times 10^{-3}$ & $10^{-1}$ & $1.5 \times 10^{-1}$ \\
\hline
\end{tabular}

2.3 "HIGH DENSITY" GAS (FOUNTAINS). The case of "high density" gas is much more complicated because radiative cooling and recombination play an important role. The borderline in density to an essentially non-cooling, fully ionized "low density" halo gas is of course somewhat uncertain, since this depends also on the history of an element of gas and the spatial distribution of heating and ionizing agents. However, the cooling shells of SNRs or, perhaps, expanding wind bubbles, certainly belong to the "high density" gas. The recombination of the gas then leads to wave damping due to ion-neutral friction (Kulsrud and Pearce, 1969) and increased diffusion of CRs through the gas. One wonders if this is not the average situation near the disk for $|\mathrm{z}|<1 \mathrm{kpc}$ (see, e.g. the review by Cesarsky, 1980). Yet, galactic fountains are overwhelmingly driven by the gas internal and dynamic pressures until radiative cooling starts to dominate the internal energy loss. Two mechanisms appear to me most likely for fountains: either the fountain is a priori ballistic, e.g. cooling SNR shell fragments continue an inertial motion upwards at already high densities and fall down subsequently. Or, hot gas wells up at somewhat lower but still "high densities" to cool, recombine, and fall down subsequently. Cosmic rays should be unimportant under both circumstances. In the first case simply due to the high inertia of such shell fragments, above everything else, and in the second still because of diffusive decoupling of CRs from gas. Thus I believe that for fountain flows the CRs are at best a nuisance, rather than a dynamical element. 
Low density, ionized gas on top of high density gas should, however, be blown away by the CRs. This should then constitute a galactic wind.

\section{GALACTIC WINDS}

3.1 MODEL ASSUMPTIONS. The first explicit theory of CR driven winds is due to Ipavich (1975). He restricted his attention to a spherically symmetric, stellar type geometry, and assumed the wave pressure to be negligible, the waves being dissipated locally and completely. The case of a plane geometry near the disk, changing over to a spherical geometry at distances exceeding the disk radius $R_{\text {gal }}=15 \mathrm{kpc}$ in a dark matter halo, and explicitly including the wave pressure, was considered by Breitschwerdt, McKenzie, and Völk $(1987,1990)$, and is briefly summarized here. The model assumptions are as follows: Only the halo dynamics is considered, with fully ionized gas, above a reference level $\left|z_{0}\right|$ of order $1 \mathrm{kpc}$. The gas is considered adiabatic - not a very critical assumption at the low reference level densities of order $10^{-3} \mathrm{H}$-atoms $/ \mathrm{cm}^{3}$ - in a 3-component flow where the gas, the CRs, and the wave field interact nonlinearly. The scattering waves are assumed to propagate outwards, amplified by the outwards streaming CRs. CR diffusion is assumed negligible in the wave frame that moves with Alfvén velocity $\underline{v}_{A}=\underline{B} /(4 \pi g)^{1 / 2}$ through the gas. Some magnetic flux tubes above the disk are open towards intergalactic space with flux tube area $A(z)=A_{0}\left(1+\left(z / z_{1}\right)^{2}\right)$, where $z_{1}=15$ $\mathrm{kpc}$, and the dynamics is considered only for those flux tubes. These flux tubes will "touch" for $|z| \gg 1 \mathrm{kpc}$ since the combined CR and wave pressures within the flux tubes exceeds the sum of gas pressure between and average field tension on flux tubes beyond such distances (Figure 2).

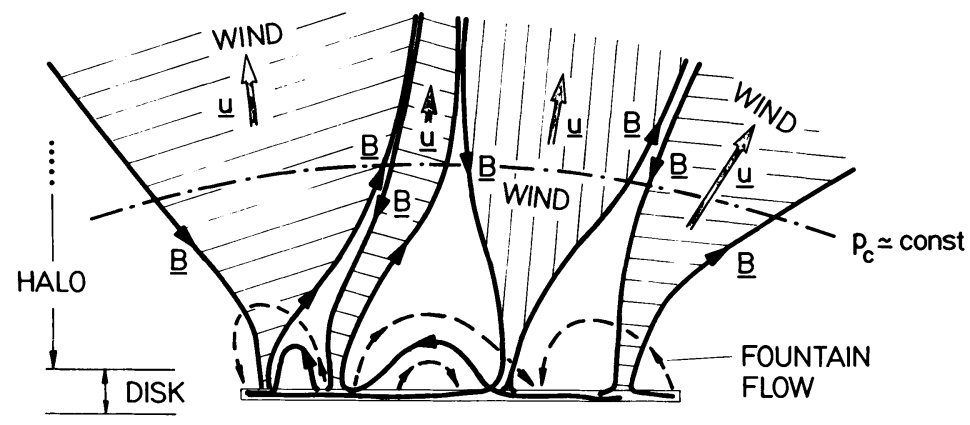

Figure 2: Schematic picture of the Galactic halo open flux tubes with CRs (hatched) which carry the galactic wind. They touch each other due to the dominance of the CR pressure $\mathrm{p}_{c}$ at greater heights. Wind speeds $\underline{u}$ can vary from one flux tube to the other. At low heights above 
the disk fountain flows and closed magnetic field lines $\underline{B}$ dominate the 3-dimensionally and magnetically structured halo.

At low $|z|$ closed field lines as well as fountain flows are abundant, but disjoint from the open flux tubes; the magnetic structure is correlated over times of order $10^{6}$ to $10^{7} \mathrm{yrs}$, the lifetime of SNRs and Superbubbles (e.g. Heiles, 1989), respectively. In such a structure the closed loops could for example be compared with those discussed by Boulares and Cox (1989). The fundamental difference is that in the model of Breitschwerdt et al. a large part of the hot gas and all the CRs exist on the open flux tubes. This relieves the closed structures of excessive particle pressure forces and, in the extreme, allows it to relax to a force free configuration. Closer in spirit is the characterisation of the halo in terms of superbubbles breaking out of the disk for which Norman and Ikeuchi (1989) invented the term "chimneys". However, I believe that also Supernovae exploding at heights $|z|>100 \mathrm{pc}$, i.e. outside the dense gas disk, should often be able to push field lines open and to initiate an outflow.

Finally, we neglect galactic rotation - a conservative assumption - and assume a steady state.

3.2 WIND SOLUTIONS WITH COSMIC RAYS. The dynamics is described by the overall momentum balance

$$
g u \frac{d u}{d z}=-\operatorname{grad}\left(p_{g}+p_{c}+p_{w}\right)-g \cdot \operatorname{grad} \phi
$$

plus appropriate energy balances for the gas, the CRs and the waves; the quantities $u$ and $\phi$ denote the mass velocity and the gravitational potential, respectively. Assuming the intergalactic pressure to be negligible the equations admit outflow solutions with a subsonic supersonic transition, i.e. supersonic winds. The square of the appropriate sound speed

$$
c_{*}^{2}=\gamma_{g} \cdot \frac{p_{g}}{\rho}+\frac{\left(M_{A}+1 / 2\right)^{2}}{\left(M_{A}+1\right)^{2}} \cdot \gamma_{c} \frac{p_{c}}{\rho}+\frac{\left(3 M_{A}+1\right)}{2\left(M_{A}+1\right)} \cdot \frac{p_{w}}{\rho}
$$

contains a sum of terms proportional to the pressures of the 3 dynamical components, where $\mathrm{M}_{\mathrm{A}}=\mathrm{u} / \mathrm{v}_{\mathrm{A}}$ is the Alfvern Mach number, $\gamma_{\mathrm{c}} \approx 4 / 3$ the adiabatic index of the CRs, and $\gamma_{\mathrm{g}}=$ $5 / 3$ that of the thermal gas. 


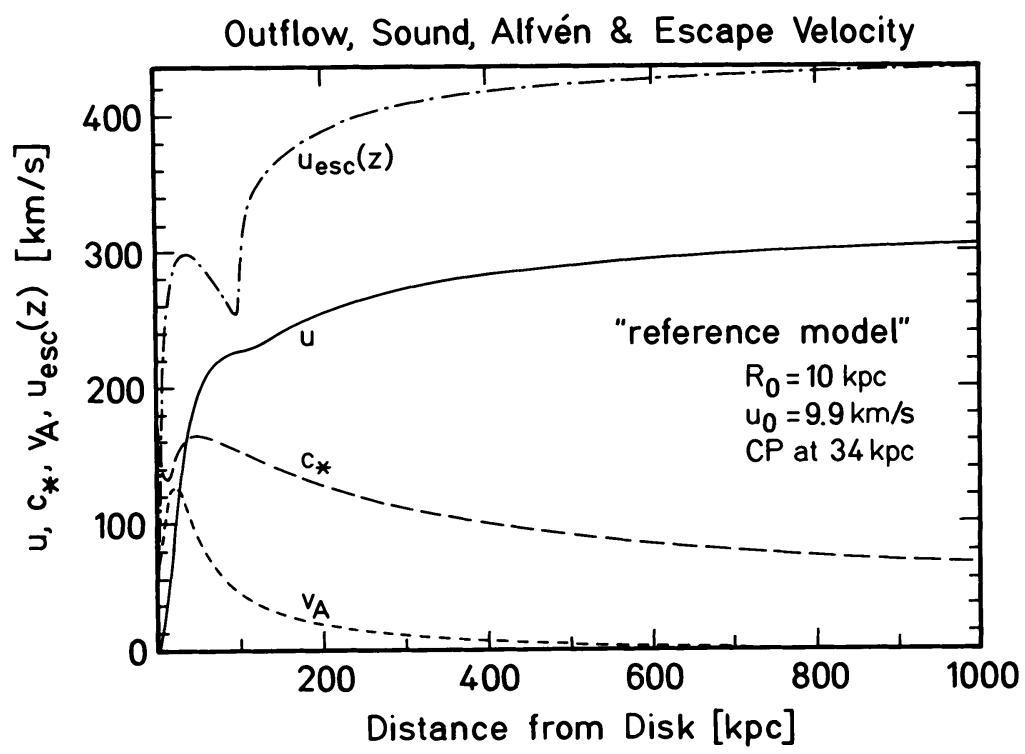

Figure 3: A plot of flow speed $u$, effective sound speed $c_{*}$, Alfvén velocity $v_{A}$, and escape speed $u_{\text {esc }}(z)$ to height $z$, as a function of the distance $z$ from the disk. The "reference model" describes a flux tube orginating at $R_{0}$ at $10 \mathrm{kpc}$ and $\left|\mathrm{z}_{\mathrm{o}}\right|=1 \mathrm{kpc}$ with $\mathrm{p}_{\mathrm{go}}=2.8 \times 10^{-13} \mathrm{dyn} / \mathrm{cm}^{2}, \mathrm{p}_{\mathrm{co}}=1 \times 10^{-13} \mathrm{dyn} / \mathrm{cm}^{2}, \rho_{\mathrm{o}}=1.67 \times 10^{-27} \mathrm{~g} / \mathrm{cm}^{3}, \mathrm{~B}_{\mathrm{o}}=1 \mu \mathrm{G}$, and a negligible wave pressure level $\mathrm{p}_{\mathrm{co}}=4 \times 10^{-16} \mathrm{dyn} / \mathrm{cm}^{2}$. The critical point $(\mathrm{CP})$, where $u=c_{A}$, lies at $34 \mathrm{kpc}$; the resulting flow speed at $\left|z_{o}\right|$ equals $u_{o}=9.9 \mathrm{~km} / \mathrm{sec}$. The kink in $u_{e s c}(z)$ occurs because the dark matter halo was assumed to terminate at $100 \mathrm{kpc}$ distance (from Breitschwerdt et al., 1990).

Figure 3 contains a representative solution for a "reference model" (Breitschwerdt et al., 1990), characterised by a flux tube foot point at $R_{0}=10 \mathrm{kpc},\left|z_{0}\right|=1 \mathrm{kpc}$. The critical point (CP) is at $|\mathrm{z}|=34 \mathrm{kpc}$, far from the disk. For the same parameters, but at $R_{0}=1 \mathrm{kpc}$, the asymptotic outflow velocity is $1500 \mathrm{~km} / \mathrm{sec}$.

3.3 GALACTIC MASS LOSS. The total energy loss, in the case of the Galaxy, is of the order of the CR energy flux from the sources of CRs. Taking the asymptotic outflow velocity as being of the order of the escape velocity $u_{e s c}$, the mass loss rate from a given flux tube is $\mathrm{M}_{\text {flux tube }} \approx \mathrm{F}_{\mathrm{co}} \cdot \mathrm{A}_{\mathrm{o}} / \mathrm{u}_{\mathrm{esc}}{ }^{2}$, where the suffix o indicates the reference level value. The total mass loss rate from the Galaxy at the present epoch is then $M_{\text {gal }}=\Sigma M_{\text {flux tubes }} \approx 1 M_{\Theta} / y r$. This rate would correspond to a mass loss over the age $t_{\text {gal }}$ of the Galaxy $\mathbf{M}_{\mathrm{gal}} \cdot \mathrm{t}_{\mathrm{gal}} \approx$ 
$10^{10} \mathrm{M}_{\odot} \cdot\left[\mathrm{t}_{\mathrm{gal}} / 10^{10} \mathrm{yr}\right] \approx \mathrm{M}_{\mathrm{gas}}$ present, of the order of the entire present ISM mass. Higher star formation rates in the past would have resulted in correspondingly higher mass loss rates.

3.4 CHEMICAL EVOLUTION OF GALAXIES. The galactic winds contain Supernovaprocessed hot gas. If single SNRs contribute to the mass loss, then the corresponding CRs, coming from the SNR's outer shock, have interstellar abundances. The CRs from superbubbles, however, where successive $\mathrm{SN}$ explosions accelerate thermal particles from the debris of the last generation SNRs, would contain an excess of heavy elements. For the gas we should, for both cases, though to different degrees, expect preferential removal of heavy elements. Thus the chemical evolution of the Galaxy should show a "slowdown" relative to naive expectations from star formation history. This appears to be indeed the case. The observed lack of heavy element enrichment in the CRs should give a limit on the Superbubble contribution to CR production, at least at the present epoch in the solar neighbourhood.

3.5 EXTERNAL GALAXIES From what has been said before, I believe that for field galaxies winds should be common, even for galaxies as relatively quiescent as ours. For starburst galaxies, like $\mathrm{M} 82$, where the gas is hot $\left(\mathrm{T}_{\mathrm{g}} \geq 10^{7} \mathrm{k}\right)$, the gas alone is able to drive a wind, as also the present theory predicts (for much earlier calculations, see Chevalier and Clegg (1985)). Then the CRs make a 10 to 30 percent contribution which is not essential. In gas-rich clusters of galaxies, on the other hand, the large intracluster pressure should inhibit winds from individual cluster members. However, generalizing our earlier arguments, we then expect a wind from the cluster as a whole.

Observations of galactic winds are severely hampered by low surface brightness. This is perhaps not too surprising for normal galaxies if we remember that the Solar Wind cannot be observed by its photon radiation even from the earth. For spectacular starburst galaxies like M 82 on the other hand, a combination of X-ray and radio continum observations shows unequivocally the existence of massive and fast winds. This has been discussed by other speakers at this symposium and therefore I will not go into it here any further except to say that M 82's mass loss is consistent with a SNR origin of the CRs (Völk, Klein, Wielebinski, 1988, 1990). Also the radio observations of closeby edge - on galaxies have been discussed in dedicated talks at the meeting (Hummel, 1990; Beck, 1990). These observations show not only good evidence for radial ( = open?) field lines in the case of NGC 4631 but also interesting $|z|$-dependencies of the polarization of the synchroton emission. Due to the spatially increasing amplitudes of the wave field, we expect a drop of the polarization at large $|z|$ from its rise from lower $|z|$. It would be interesting to verify such an expectation for a sample of nearby edge-on galaxies.

It seems that we will not have to wait too long to get these observations. Then galactic winds, even from normal galaxies, will be more than a compelling theoretical prediction. The consequences for the mass and energy balance of the extragalactic medium need to be investigated in the future. 
ACKNOWLEDGEMENTS. I would first of all like to thank D. Breitschwerdt and J.F. McKenzie for the continued collaboration on galactic winds we enjoy together. My thanks are also due to R. Beck, C.J. Cesarsky, M. Dougherty, E. Hummel, and C.M. Ko for discussions on various aspects of this paper.

\section{REFERENCES}

Beck, R. (1990) these proceedings

Boulares, A., Cox, D.P. (1989) Ap. J. submitted

Bregman, J.N. (1980) Ap. J. 236, 577

Breitschwerdt, D., McKenzie, J.F., Völk, H.J. (1987) in "Interstellar Magnetic Fields", eds. R. Beck, R. Gräve, Springer Heidelberg, p. 131

Breitschwerdt, D., McKenzie, J.F., Völk, H.J. (1990) submitted to Astron. Astrophys.

Cesarsky, C.J. (1980) Ann. Rev. Astron. Astrophys. 18, 289

Chevalier, R.A., Clegg, A.W. (1985) Nature 317, 44

Cox, D.P. (1981) Ap. J. 245, 543

de Boer, H. (1990) these proceedings

Dougherty, M.K., McKenzie, J.F., Westergaard, N.J. (1985) Proc. 19th. Int. Cosmic Ray Conf., La Jolla 3, 83

Drury, L. O'C., Markiewicz, W.J., Völk, H.J. (1989) Astron. Astrophys. 225, 179

Ginzburg, V.L., Ptuskin, V.S. (1985) Sov. Sci. Rev. E. Astrophys. Space Phys. 4, 161

Habe, A., Ikeuchi, S. (1980) Prog. Theor. Phys 64, 1995

Heiles, C. (1984) Ap. J. Suppl. 55, 585

Heiles, C. (1989) in Proc IAU Coll. No. 120. eds. G. Tenorio-Tagle, M. Moles, J. Melnick, Springer, Heidelberg, p. 484

Hummel, E. (1990) these proceedings

Ipavich, F. (1975) Ap. J. 196, 107

Kahn, F.D. (1981) in "Investigating the Universe", eds. F.D. Kahn, Reidel, Dordrecht, p. 1

Kennicutt, R.C. Jr., Edgar, B.F., Hodge, P.W. (1989) Ap. J. 337, 761

Ko, C.M., Dougherty, M.K., McKenzie, J.F. (1990) Astron. Astrophys. in press

Kulsrud, R.M., Pearce, W.D. (1969) Ap. J. 156, 445

Lockman, F.J., Hobbs, L.M., Shull, J.M. (1986) Ap.J. 301, 380

Markiewicz, W.J., Drury, L. O'C., Völk, H.J. (1990) Astron. Astrophys. in press

Mathews, W.G., Baker, J.C. (1971) Ap. J. 170, 241

Mirabel, I.F. (1989) in Proc. IAU Coll. No. 120, eds. G. Tenorio- Tagle, M. Moles, J. Melnick, Springer, Heidelberg, p. 396

Norman, C.A.,Ikeuchi, S. (1989) Ap. J. 345, 372

Shapiro, P.R., Field, B.G. (1976) Ap. J. 205, 762

Völk, H.J., Klein, U., Wielebinski, R. (1988) Astron. Astrophys. 213, L2

Völk, H.J., Klein, U., Wielebinski, R. (1990) to appear in Astron. Astrophys. 


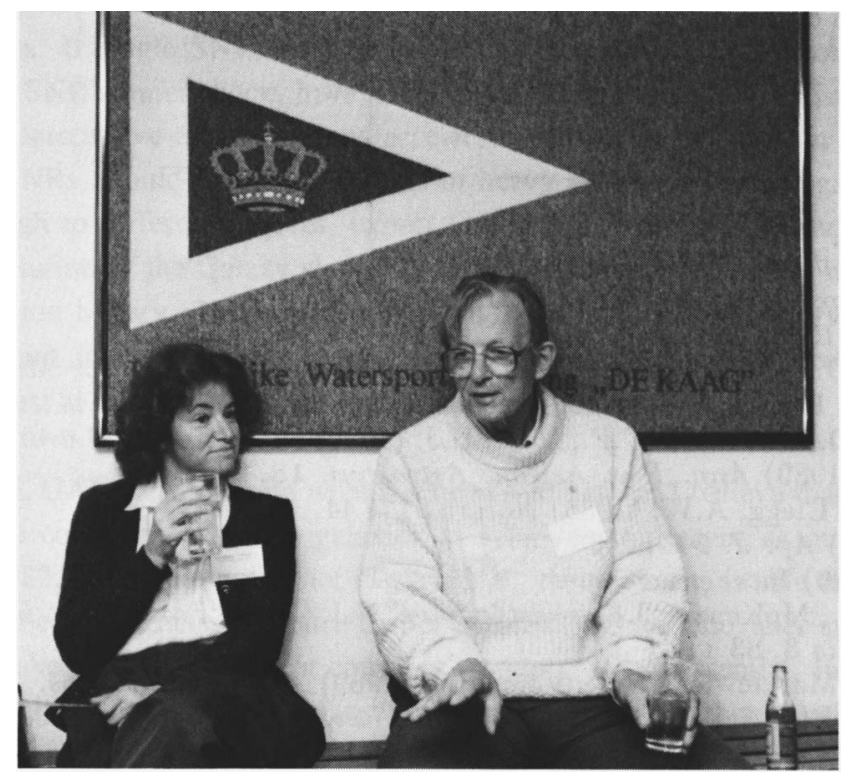

